\title{
Factors that may influence the performance of wireless sensor networks
}

\author{
Majdi Mansouri*, Ahmad Sardouk, Leila Merghem-Boulahia, \\ Dominique Gaiti and Hichem Snoussi* \\ ${ }^{*} I C D / L M 2 S, I C D / E R A$, UMR 6279, Troyes University of Technology
}

France

\author{
Rana Rahim-Amoud \\ LaSTRe, Université Libanaise \\ Lebanon
}

Cédric Richard

Laboratoire FIZEAU UMR CNRS 6525 Université de Nice Sophia-Antipolis

France

\section{Introduction}

The Wireless Sensor Networks (WSNs) are penetrating more and more our daily life. They are used in a large type of applications as supervision, tracking and control in military, environmental, medical and several other domains. Therefore, new approaches and protocols are proposed every day in order to optimise the performance of the WSNs and to increase their reliability and quality of service. These new protocols take into consideration the challenges of the WSN and they are built up some key factors (parameters and concepts) to achieve their goals.

The aim of this chapter is to study the factors that may influence the desired performance of the WSNs. These factors are inspired from the sensor nodes characteristics, the physical deployment of the WSNs and the WSNs' information functions. Firstly the sensor nodes are characterized particularly by their limited power and memory capacities. The power is used to be a key parameter for any approach supposing that sensor nodes' batteries are unchangeable and not rechargeable. The power would influence the reliability of the network, if the residual battery of an important node, as a cluster head, is limited. Respecting the residual battery of the node leads to a more efficient routing path, cluster head designation, aggregation point selection, etc. The limited memory is also a very important parameter as it defines the size of the operating system and the processing code. It also defines the amount of information that a node is able to store. For example, this parameter has to be managed in a mobile sink Chatzigiannakis et al. (2008) Cheng et al. (2009) approach, to define the duration that a node may tolerate before communicating its information to the sink and to optimize the mobile sink trajectory. 
Secondly, the physical deployment of the WSN has to be studied to satisfy the application requirements. The network deployment identifies the density of the network. A random deployment may lead to different density levels in the network. Thus, the redundancy level will not be the same in the entire network, also a sleeping decision of a node in a dense zone will not have the same influence as in a sparse zone. The density could also be correlated with the sensing coverage of the nodes and the global covered area. The lower is the sensing coverage, the higher is its precision level. For example, in a bordure supervision application, the sensing coverage and the density should be combined to minimize the probability of having vulnerable zones. The random deployment leads also to a different nodes position. Thus, a node connecting two parts of the network has to be always activated to minimize, for example, the end-to-end communication delay or to insure a higher connectivity of the network. The position of a node within the WSN may optimise the definition of its role (aggregator, normal, cluster head) and main operation (routing, perception).

Thirdly, the radio communication defines several parameters as the transmission power, the signal to noise ratio and the radio coverage. The radio communication is known to be the main source of power consumption in WSN. Thus, higher is the transmission, shorter is the sensor node lifetime. However, the variable transmission power could be a good solution in a cluster based approach, where the members limit their transmission power to reach their cluster head and this latter will use a higher one to reach its neighbor's cluster heads. The signal to noise ratio could be also investigated to select an aggregator node in a zone with the higher ratio to avoid the estimation error. This ratio could be also used to avoid the radio communication interference in dense zones of the network. The last parameter is the radio coverage, which insures that the supervised area is completely covered and the deployment of new nodes will not lead to the creation of isolated networks. It is also a parameter that may define the necessity of deploying new sensor nodes.

Thirdly, the information is certainly the goal of the WSN deployment. Therefore several methods exist to estimate the relevance of the gathered information, to estimate future information and to eliminate the redundancy. Thus, in this chapter, we discuss some of the parameters and the models that are used to determine the importance of the information and to estimate it in order to optimize the end-to-end delay.

The remaining of this chapter will discuss, in section 2, the sensor nodes characteristics and their possible influence on the WSN performance. Then, in section 3, we discuss the impact of the network deployment on the accuracy of the gathered data and on the optimal WSN lifetime. Next, in section 4, an analytical study is giving about the sensor nodes' information characteristics, in terms of relevance and prediction. Finally, the conclusion is given, in section 5 .

\section{Sensor nodes' characteristics}

The hardware capacities of the sensor nodes define the limits of any application or optimization proposal in WSN. Indeed, the algorithms that they are not limited by the CPU, memory, radio communication or power constraints could lead to a high performance in terms of realtime communications and successful data delivery and precision. However, the main challenge in WSNs is the limited hardware capacities. Thus, in this section, we discuss the general types of the sensor nodes and we present their actual technologies advancement. 


\subsection{Sensor nodes' types: a classification by application nature}

The WSNs are penetrating our daily life in several kinds of applications such as military, environmental, health, habitat, industrial, etc. Indeed, multitude types of sensor nodes equipment with various capacities and goals are proposed to achieve the requirement of theses daily applications. These types could be classified up on the nature of the applications Yick et al. (2008). In the remains of this section, some sensor nodes' types will be discussed based on their application requirements.

For large scale environmental applications as in forest, desert or normal natural conditions, Terrestrial Sensor Nodes (TSNs) Yick et al. (2008) Akyildiz et al. (2002) could be deployed. The TSNs are supposed to be inexpensive and deployed in hundreds to thousands in an area of interest. They could be deployed randomly as threw by plane or placed in pre-planned positions Stavros \& Leandros (2006) Pompili et al. (2006) by humans or robots. These sensor nodes are self organized; they built up autonomously the network connection and communicate in a multi-hop manner. The TSNs have to communicate efficiently their environmental measures back to a base station. However, their limited batteries could be a big challenge. To the best of our knowledge, the communication is the main consumer of the power in sensor nodes, while the TSNs' batteries could be unchangeable due to, e.g., the hazardous zone of the deployed nodes. Therefore, several approaches have been proposed to reduce the power consumption of the sensor nodes: (1) optimizing the data communication routes to be shorter and energy-aware Ok et al. (2009) Chang \& Tassiulas (2004), (2) defining optimal duty cycles within an energy-aware Mac layer Ye et al. (2002) Polastre et al. (2004), (3) reducing the number of communication sessions and the amount of communicated data by applying efficient data aggregation methods Sardouk et al. (2011) or even (4) attaching secondary batteries or solar charger to the sensor nodes.

In special industrial applications, such as underground mine or petroleum fields, more physically powerful sensor nodes are needed. Thus, the UnderGround Sensor Nodes (UGSN) Ian F. \& Erich P. (2006) Li \& Liu (2007) are supposed to be more expensive than the TSNs as they had to ensure reliable communication through soil, rocks, water and other mineral contents. The UGSNs' deployment is application tailored and it could not be generalized. Also, the network maintenance and post deployment are expensive and quite difficult due to the nature of the monitored mine or cave. In addition, there is a high probability of communication problems as signal loss and high level of interference and attenuation caused by the nature of the environment.

Similarly to TSNs, the UGSNs have strict power constraints as their battery could be unchangeable or unchargeable. Thus a power aware network deployment, communication, and data aggregation had to be studied.

The UndeWater Sensor Nodes (UWSN) are designed to be deployed in underwater applications Heidemann et al. (2006). Indeed, due to the underwater conditions, these sensor nodes are supposed to be more expensive than the TSNs and somehow less expensive than the UGSNs. However, the underwater WSNs applications are not supposed to be as dense networks. The typical challenge of the (UWSN) is the acoustic communication problem as the high propagation delay, the limited bandwidth and the signal fading. Moreover, the acoustic conditions increase the sensor nodes failure, which leads to serious network partitioning and data loss. 
Here also, the UWSNs are similar to TSNs and UGSNs in terms of power constraints and impossibility of battery charging or replacing.

Another type of senor nodes that could be distinguished is for the multimedia applications Yick et al. (2008) Akyildiz et al. (2007). Thus, we call them as MSNs (Multimedia Sensor Nodes). They could be similar in physical forms to any one of the above mentioned types (TSN, UGSN and UWSN). However, the MSNs have, in addition, a built-in or attached cameras and they may require more powerful processing and storage units as they are supposed to communicate captured images, videos and/or sounds to a base station. Due to their nature, the radio entity of the MSNs should have some special specifications to ensure a minimal quality of service (QoS) level. The required QoS could be also influenced by the sensor node processor that may need to execute some image processing or compression before sending the results to a base station. However, the MSNs' deployment is generally pre-planned to ensure the aimed coverage level.

The TSNs, UGSNs, UWSNs and the MSNs could be fixed or mobile nodes. Indeed, the mobility could be an important issue as it may permit a better event or interest centric deployment. It offers a deeper and wider exploration of the area of interest. In terms of energy, the mobile sensor nodes are certainly more consumer, in order to supply the movement engine. However, they could be more efficiently chargeable throw sun panels as they could move to a better sun exposure.

The mobile and fixed TSNs, UGSNs, UWSNs and the MSNs could be used in numerous civil, military and industrial applications. In the above discussion, two main challenges could be pointed out. The first one is the limited power of the sensor nodes and the second challenge is the required reliable communication in various condition (underground, underwater, with QoS, etc.) The multimedia WSNs define also the importance of the processing and storage capacities. However, the optimization, in terms of power and communication, passes generally through algorithms as softwares for the application layer, or protocols for the transport, network or Mac layers. Indeed, more powerful are these algorithms, more the power and the communication are optimized. Thus, the processing unit capacity could also be a key factor in any optimization proposal for the WSN.

In the next section, a brief discussion of today technologies advancement in terms of processors speeds, memory storage and power consumption is presented.

\subsection{Technologies advancements}

In our days, TinyOs Hill et al. (2000) and sunSPOT Sun (2008) seem to be the most important technologies of wireless sensor nodes. The first one is a simple, lightweight event-based operating system written in nesC Gay et al. (2003) that is widely spread (it is used on Crossbow motes, Moteiv motes and similar devices).

The second, sunSPOT, is a product of Sun Microsystems, Inc. encompassing both hardware and software Sun (2008). The project started in 2003 on the experience of the company with the technologies related to java ME, and the first released occurred in April 2007. The recent release of platform Platon \& Sei (2008) entails that the hardware provides among the most powerful sensor nodes, with similar size and scale factors of motes. The software part is independent from the hardware and consists of the Sun Squawk Java virtual machine Sun (2008). 
Squawk is a closed-source JVM that encompasses necessary operating system functionalities, so that it can run directly on hardware Shaylor et al. (2003).

The remains of this section presents the hardware capacity of these technologies and a comparison with other technologies.

\section{Hardware}

A sensor node is made up of five basic entities: sensors, processor, memory, radio, and power entity. They may also Akyildiz et al. (2002)have application dependent additional components such as location finding system, a power generator and a mobilize.

Sensors are electronic devices that are capable to detect environmental conditions such as temperature, sound, chemicals, or the presence of certain objects. They send detected values to the processor which runs the sensor operating system and manages the procedures required to carry out the assigned sensing task. This processor retrieves the application code from the memory unit which stores also the operating system and the sensed values.

The radio permits to the wireless sensor nodes to communicate with other nodes, to receive commands and updates from the sink and to send sensed data to the sink.

The key element in a sensor node is the power entity which is generally composed of a couple of standard AA batteries. The size of these batteries usually determines the size of the sensor. Further, studies Baronti et al. (2007) are currently under way to replace/integrate battery sources with some power scavenging methods such as solar cells. In fact, there are some limits about the actual effectiveness of such methods. For example, solar cells do not produce much energy indoor or when covered by tree foliage.

In table 1 we compare some important sensor nodes such as Micaz, sunSPOT, TelosB, Sentilla and IMote2. The first three rows represent their processing and storage capacities, where the remaining rows represent their power consumption in three different cases: idle, active, and sleep. As shown in this table TelosB and Sentilla consume the least but they are very limited in term of storage and processing compared to Micaz, sunSPOT and IMote2. On the second hand, sunSPOT and IMote2 are the most powerful in terms of processing and storage but in the same time they consumes a lot of power. We can mention also that IMote2 has the biggest story capacity, which is due to its utilization in multimedia WSN. Hence, the IMote2 nodes are supposed to store captured images, videos and/or sounds that may require high a relatively high large space.

\begin{tabular}{|l|c|c|c|c|c|}
\hline & MICAZ(Crossbow) & SunSPOT & TelosB & Sentilla & IMote2 \\
\hline Processor (Mhz) & 16 & 180 & 8 & 8 & $13-416$ \\
Ram (kb) & 4 & 512 & 10 & 10 & 256 \\
Flash (KB) & 512 & 4096 & 1024 & 48 & 32000 \\
Active draw (ma) & 48 & 70 & 25 & 4 & $>44$ \\
Idle draw (ma) & 8 & 24 & 2 & 1 & $>31$ \\
Sleep draw ( $\mu \mathrm{a})$ & 15 & 32 & 6 & 1 & 387 \\
\hline
\end{tabular}

Table 1. Sensor nodes features 
Based on this comparison, Micaz appears to be a combination between the first two nodes and the sunSPOT and IMote2. It consumes less then the powerful nodes and it is more powerful in processing and memory storage than TelosB and Sentilla.

\subsection{Radio entity: importance and power consumption}

The radio communication entity of the sensor node is certainly, the main entity to build the wireless network. This entity is known to be the main power consumer of the node. However, this consumption is due to achieve an acceptable level of reliable communication. In this section, we will illustrate that by showing the importance of the high transmitting power.

Let suppose a network divided into clusters, hence the cluster members will communicate their data to the cluster head $(\mathrm{CH})$. Thus, the total amount of required transmission power used by the $i$-th sensor within a cluster Cui et al. (2005) is proportional to:

$$
\begin{aligned}
P^{i}(t) & \propto d_{i}^{\lambda}\left(N_{t}^{i}-1\right) \\
& \equiv\left\|s^{i}-L_{C H_{t}}\right\|^{\lambda}\left(N_{t}^{i}-1\right)
\end{aligned}
$$

where, $d_{i}$ is the transmitting distance (meters) between the $\mathrm{CH}$ and the $i$-th sensor, $L_{\mathrm{CH}_{t}}$ is the location of the $\mathrm{CH}$ at the sampling instant $t$ and $\lambda$ is the path loss exponent.

The importance of the high transmitting power could be illustrated in figure 1.This figure presents the average distance estimation error versus transmitting power, in a tracking application using the variational filtering (VF) based on quantized proximity sensors Mansouri et al. (2009) (see section 4). In the X-axis, we change the transmitting power of the sensor node. Then, on the Y-axis, we observe the influence of the transmitting power on the separating (between target and sensor node) distance estimation. Thus, we can mention that in low transmission power, the distance estimation error (RMSE) is at its higher value (around 6 meters). However, by increasing the transmission power, the RMSE become lower. We can observe also that, after a certain value, the transmitting power could be optimized and there is no need to choose higher values. Power consumption of the radio entity

The amount of energy consumed in a communication could be computed Sohraby et al. (2007) by equation 2, where $E_{T X}$ is the power consumed during the transmission and $E_{R X}$ is the power consumed during the reception. Both of them are computed following the data length and transmission distance (radio range of the node) $(1, \mathrm{~d})$;

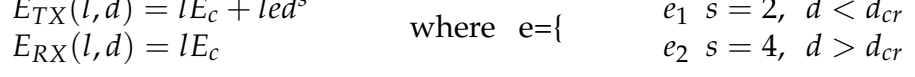

Where $E_{c}$ is the base energy required to run the transmitter or receiver circuitry. A typical value of $E_{c}$ is $50 \mathrm{~nJ} /$ bit for a $1-\mathrm{Mbps}$ transceiver; $d_{c r}$ is the crossover distance, and its typical value is $87 \mathrm{~m}$; $e_{1}\left(e_{2}\right.$ respectively) is the unit energy required for the transmitter amplifier when $\mathrm{d}<d_{c r}$ (or $\mathrm{d}>d_{c r}$ respectively). Typical values of $e_{1}$ and $e_{2}$ are $10 \mathrm{pJ} / \mathrm{bit} . \mathrm{m}^{2}$ and $0.0013 \mathrm{pJ} /$ bit. $\mathrm{m}^{4}$, respectively. 


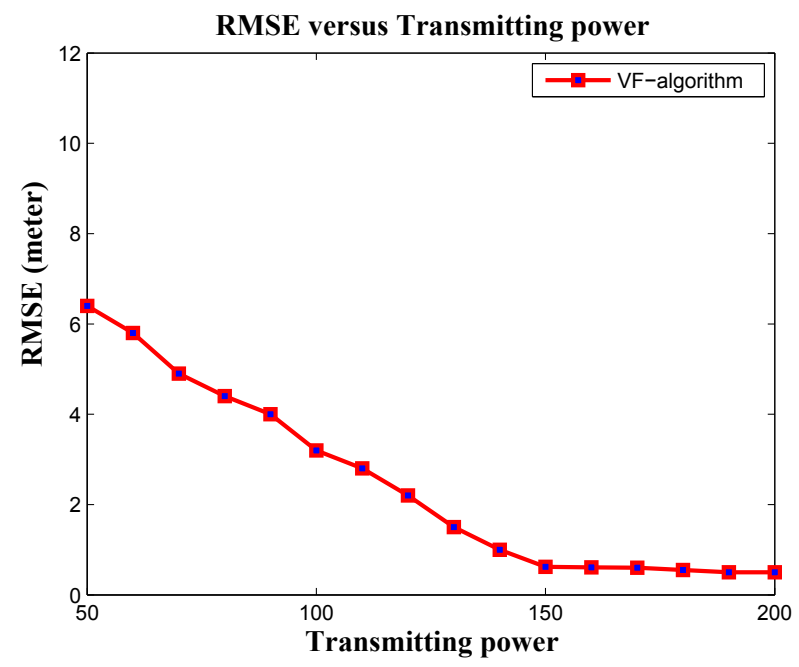

Fig. 1. RMSE vs Transmitting power varying in $\{50, \ldots, 200\}$.

\section{Impact of network deployment on data credibility}

The data accuracy is one of the key factors for an efficient data aggregation in WSNs. Thus, the aggregated data need to represent, geographically, the maximum possible of the monitored area. Hence, several points related to the network deployment have to be adapted to have data representing the whole monitored area. Some of these points are as follows:

- Mainly the deployment model: In an accessible and small area, the sensor nodes could be placed one by one to insure a high representability of the monitored area. However, in large and inaccessible zones, the sensor nodes are supposed to be randomly deployed. Hence, the nodes could be grouped in some places while others are not covered;

- The network density: It represents the number of sensor nodes per square meter. This point could be easily managed in manual placed nodes. However, it seems to be difficult to manage in case of randomly deployed WSNs;

- The sensing coverage per sensor node: I.e. each node is supposed to represent a circle centered on it and with a radius $r$ defined by the system developer.

The density and sensing coverage could impact together or separately the determination of the covered area, which could be illustrated in figure 2 .

In figure $2 \mathrm{a}$, the density is low and the sensing coverage is limited, hence the non covered surface (gray color) are important. By keeping the density low and enlarging the sensing coverage (figure $2 b$ ), the non covered area is reduced. Similarly, it is possible to increase the covered area, by keeping the sensing coverage limited and increasing the density (figure 2c). 

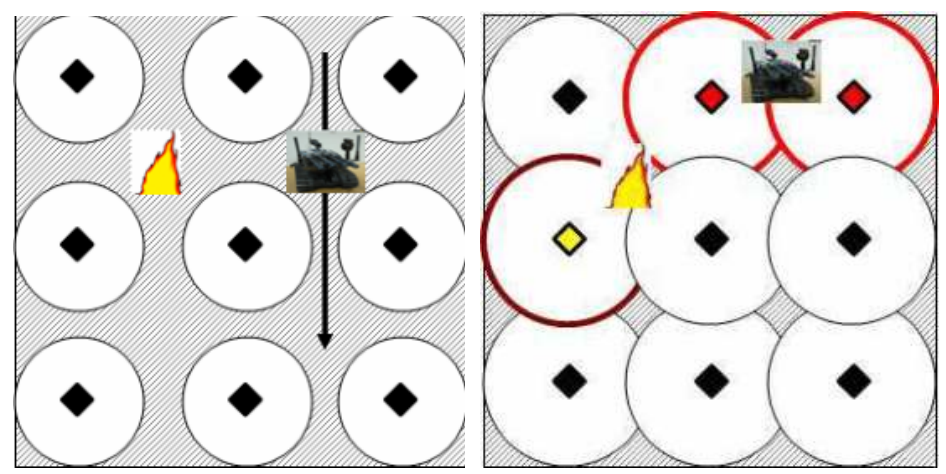

(a) Low density reduced sensing cov- (b) Low density large sensing covererage age
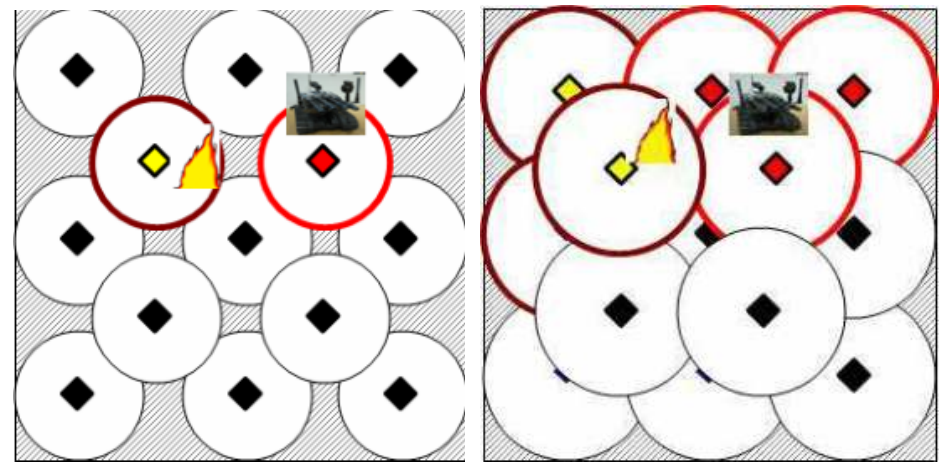

(c) High density reduced sensing (d) High density large sensing covercoverage age

\section{- Sensing coverage per sensor node \\ $\Lambda$ Event \\ object}

Fig. 2. Impact of density and sensing coverage

Finally, by enlarging the sensing coverage and increasing the density (figure 2d), the non covered area could be reduced more and more.

The impact of the network deployment, in manual placed nodes, on data accuracy, is out of the scope of this section, as it is supposed to be influenced by the choices of the system developer. Thus, this section discusses the case of randomly distributed WSNs. The current analysis is based on the comparison between low density, and high density WSNs, with a variation of the sensing coverage per node. The comparison includes three types of random 
deployment, which are as follows:

- Uniform random distribution where all the nodes have equal probabilities to be placed in any position in the area;

- Column-based random distribution: It divides the network area into approximately equal columns. Then, it distributes the nodes randomly in each column. This type pf deployment is supposed to be closer to the reality than the first one;

- Grid-based random distribution: It divides the network area into approximately equal columns and rows. Then, it distributes the nodes randomly into the obtained cells. It is more complicated than the two others, however it is very probable in real applications.

For each one of these deployment methods, the current analysis discusses the distribution of the nodes and the percentage of the covered area regarding the whole monitored area.

\subsection{Low density WSNs}

Figure 3 illustrates the deployment of 200 sensor nodes in an area of $1000 \times 1000 \mathrm{~m}^{2}$. Figure $3 \mathrm{a}$ shows that, in a uniform random distribution, the density is high in the southeast quarter of the area, while it is very low in the northwest quarter. In the two other quarters, it is uniform. That means that the northwest quarter's data are not well represented while in the southeast quarter there is a redundancy in the data due to the correspondent density of nodes. Figure $3 b$, presents that in column-based random distribution the monitored area is better covered compared to the uniform random distribution. However, some zones are still better represented (south part) than others (north part). The Grid-based random distribution, Figure 3c, offers the best deployment where there is somehow an equitable representation of the monitored area.

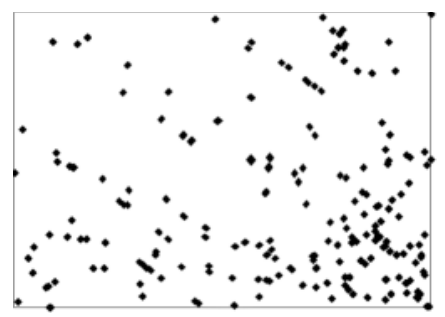

(a) Uniform random distribution

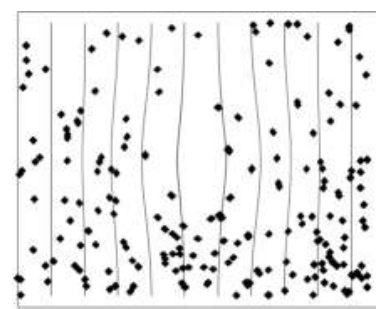

(b) Column-based random distribution

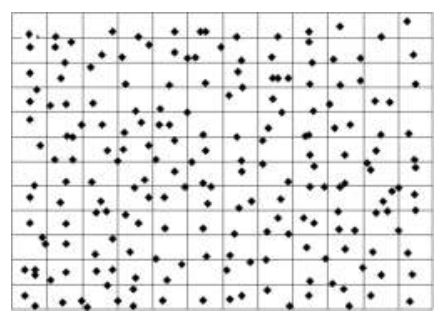

(c) Grid-based random distribution

Fig. 3. Impact of nodes deployment on data accuracy in low density WSN

\subsection{High density WSNs}

Figure 4 illustrates the deployment of 800 sensor nodes in the same area $\left(1000 \times 1000 \mathrm{~m}^{2}\right)$. Figure 4a shows that, in a uniform distribution, the density is high in the northwest and southeast sides of the area, while in the middle and borders it is low. In a column-based 
distribution (figure $4 \mathrm{~b}$ ), it is much better except in the middle of the area, where it is not so representative. The grid-based method (figure 4c) distributes again equitably the sensor nodes over the monitored area.

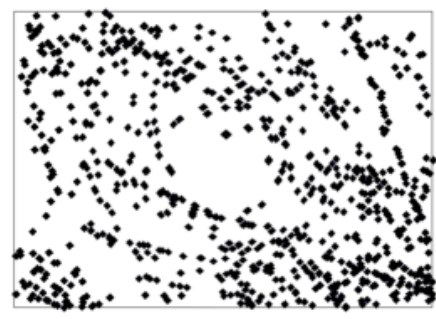

(a) Uniform random distribution

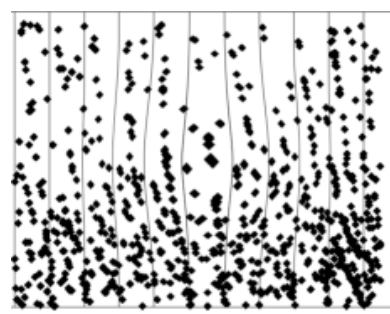

(b) Column-based random dis- (c) tribution

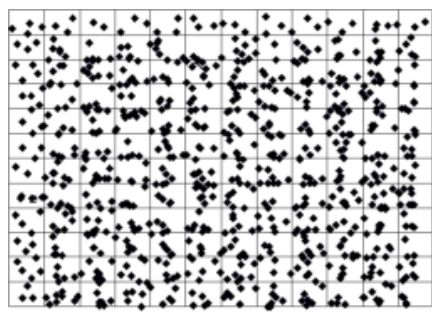

(c) Grid-based random distribution

Fig. 4. Impact of nodes deployment on data accuracy in high density WSN

\subsection{Network density}

The network density is the number of nodes per square meter. It varies from one deployment to another and from one node to another within the same deployment depending on the node distribution.

According to Akyildiz et al. (2002), this parameter does not have a fixed value to be used as a reference. The ideal value is application and environment dependent. In addition, this parameter has a network management importance as it helps to identify the dense zones of the network and the non well covered zones. Hence, it may lead to redeployment of more nodes in some zones for a better coverage.

We propose that each sensor node computes its own network density. There are two main reasons behind that: the first one is that each node has its dedicated view of the network (which is limited to its neighbors). The second and most important reason is the fact that for a specific task, the needs for a cooperation is between the sensor nodes of the same zone (geographical part of the network) and not farther nodes. For simplicity's sake, we propose the equation (3) to compute this density (D). In this equation, we compute the percentage of the real density compared to the theoretical density (both of them are explained later on), i.e., a density bigger than $100 \%$ could exist in the case of a very dense zone (in this case, the tendency of the sensor node will be toward the selfishness, hence to preserve its battery). It is important to note here, that if the density is greater than $200 \%$ it will be limited to this value to avoid an overweight estimation of density. Otherwise, if the density computed by a node is equal to $0 \%$, it means that for example the node is disconnected from the network. 


$$
\begin{array}{cl}
\mathrm{D}= & \frac{\text { realdensity }(R D)}{\text { theoreticaldensity }(T D)} \\
\text { where, } & \\
\mathrm{RD}= & \frac{N_{\text {real }}}{\left(\pi \times r^{2}\right)} \\
\text { and, } & \\
\mathrm{TD}= & \frac{N_{\text {theoretical }}}{\left(\pi \times r^{2}\right)} \\
\text { hence, } & \\
\mathrm{D}= & \frac{N_{\text {real }}}{N_{\text {theoretical }}}
\end{array}
$$

Where $\mathrm{r}$ is the radio range of the sensor node, $N_{\text {theoretical }}$ is the theoretical number of nodes and it is given from the ideal distribution of the nodes or the grid distribution (figure 5a). $N_{\text {theoretical }}$ corresponds to the number of nodes within the radio range of a reference node (RN). A RN is a node in the center of the area to eliminate the special cases of border nodes.

$N_{\text {real }}$ is the number of the one hop neighbor nodes, appearing on the neighbors or routing table of the node in question. $N_{\text {real }}$ should be equal to $N_{\text {theoretical }}$ in the ideal case. Figure $5 \mathrm{~b}$ shows an example of randomly distributed nodes to give an idea about real network densities.

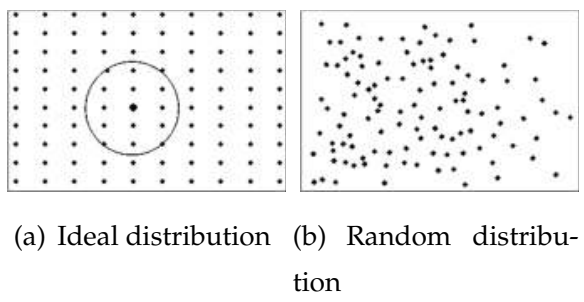

Fig. 5. Network density comparison

\section{Impact of network density on multi objects tracking}

Figures 6 and 7 compare the performance of two multi objects tracking algorithms (PF Djuric et al. (2003) and QVF Mansouri et al. (2009)) in a sparse and dense WSNs, respectively. figures $6 \mathrm{a}$ and $7 \mathrm{a}$ present the behavior of both algorithms, in tracking the two targets in question. We can observe that both algorithms behave better when the network is dense. That is due to fact that, in dense networks, the number of nodes detecting the object is higher; therefore the estimation of its position and its next position is more reliable.

figures $6 b, c$ and $7 b, c$ presents the distance estimation error in both algorithms, for WSNs of 400 nodes and 800 nodes, respectively. In low density network (figure $6 \mathrm{~b}, \mathrm{c}$ ), the distance estimation error in VF is in average variable between 0 and 1 meter. On the other side, in higher density (figure $7 b, c$ ), the distance estimation error is in average divided by 2 , were it is approximately less than 0.5 meters. For PF, the optimization is similar, where the errors in low density (figure $7 \mathrm{~b}, \mathrm{c})$ ) are around 4 meters. Then, these values are approximately divided by two when the network density is increased (figure $7 b, c)$ ), thus the distance estimation error become less than 2 meters. 


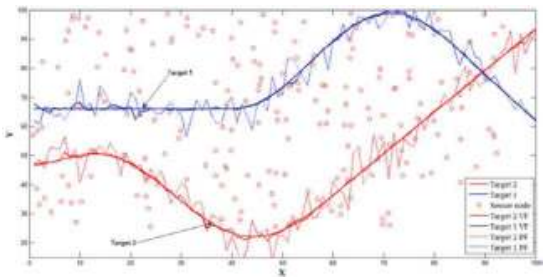

(a) Two objects tracking

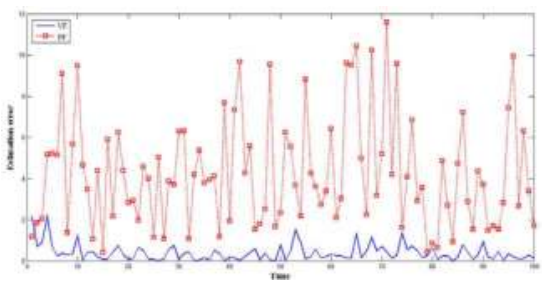

(b) 1st object : error estimation

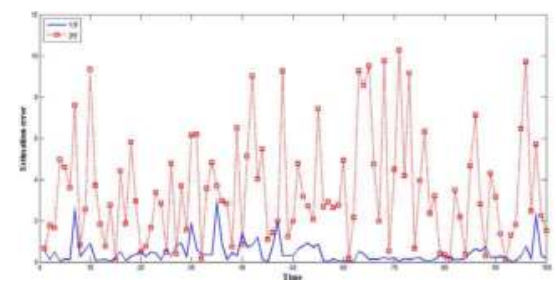

(c) 2nd object : error estimation

Fig. 6. Multi objects tracking in low density network, 400 nodes

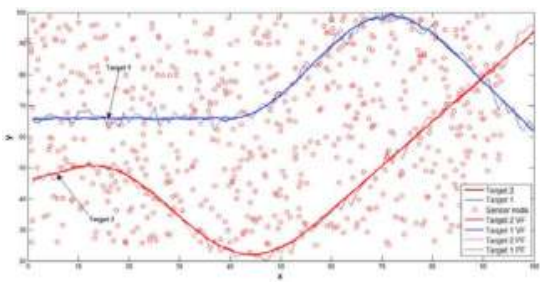

(a) Two objects tracking

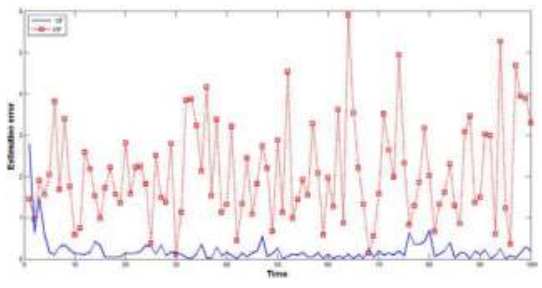

(b) 1st object : error estimation

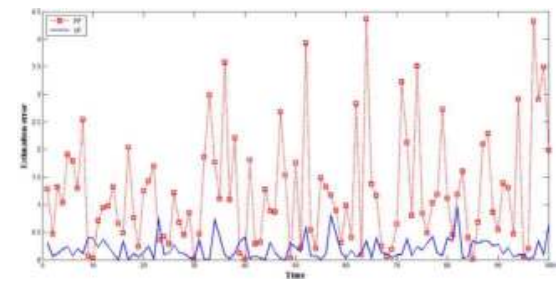

(c) 2nd object : error estimation

Fig. 7. Multi objects tracking in high density network, 800 nodes 


\subsection{Node position within the network}

Another parameter related to the network deployment has also to be studied carefully, due to its importance as we ill explain in this section. This parameter is the position $(\mathrm{P})$ of the agent node in the network. We define three types of node positions: (1) normal, (2) edge and (3) critical. The normal position is the position inside the network where the node has multiple neighbors. This kind of nodes may tend toward the cooperative behavior, to maximize the amount of the important information collected in the network. The edge node ( $\mathbf{E}$ in figure 8) is a node in the border of the network, which has a restricted view of the network limited to only one neighbor.

A node is considered in a critical position (C in figure 8) if it connects two parts of the network. That means, if the node runs out of battery, it may divide the network and multiple nodes behind it will become unreachable and in the best case they will require a longer route to communicate their data to the sink. This longer route is expensive in term of energy as the number of hops is increased. For example, in figure 8 , if a $\mathbf{C}$ node runs out of battery, the network will be divided in two parts.

A good strategy should allow a sensor node in a critical position to decrease its power consumption to maintain the connection between the two parts of the network the longest possible time. Thus, the value of the importance factor of the node position should help the sensor node to apply a selfish behavior and hence, e.g, it should be greater than or equal to the energy or the information importance degree factors.

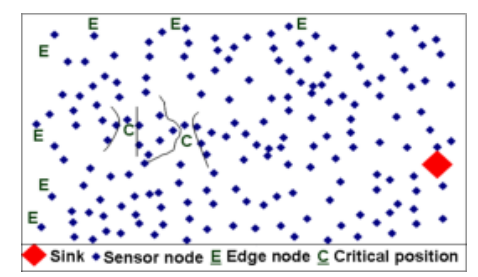

Fig. 8. Nodes' positions in the network

To facilitate the computation of $\mathrm{P}$, we propose a fixed value for each type of node position. These values are $10 \%, 50 \%$ and $100 \%$ for the normal, edge and critical, position respectively.

\section{Mean time before first partitioning}

The mean time before first partitioning (MTBFP) in WSN, could be measured by the occurred duration before the loss of the first critical node. Thus, in Sardouk et al. (2011), we study a data aggregation method that takes into consideration the position of the sensor node. This method is simulated in two scenarios. The first one takes into consideration the position of the sensor nodes during the data aggregation (IIBC+P). The second scenario (IIBC) supposes that all the sensor nodes are equals.

In figure 9, we present a comparison, in terms of power consumtion, between the both scenarios IIBC and IIBC $+\mathrm{P}$. As we can observe, IIBC $+\mathrm{P}$ decreases the average power consumption 
of the critical sensor nodes in an important manner. It shows also that more the network is dense more the amount of decreased power is relevant. We can also observe that for 500 nodes, IIBC $+\mathrm{P}$ divides by more than 5 the consumption of these nodes compared to IIBC and for 700 and 900 nodes, this optimization remained important where IIBC+P divides the consumption by more than 4 . In addition, in a non dense network, the power consumption has been divided by a factor of approximately 3 .

Hence, we can deduce from these curves that IIBC $+\mathrm{P}$ offers a better power management for nodes in critical positions independently from the network scale and density.

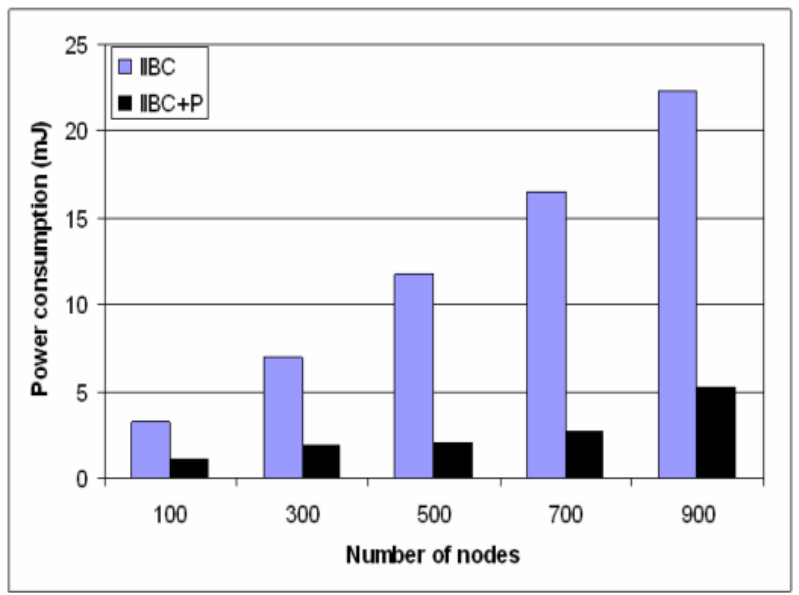

Fig. 9. Average power consumption per node in critical position

\section{Information relevance: a study}

The information relevance parameters computing is done following the model proposed in Mansouri et al. (n.d.); in which we assume that: i) the sensor measurements are quantized before being transmitted (a quantized proximity sensors is considered), ii) the application is the target tracking.

\subsection{Quantized Observation Model}

Consider a wireless sensor network, in which the sensor locations are known $s^{i}=\left(s_{1}^{i}, s_{2}^{i}\right)$, $i=1,2, \ldots, N_{s}$. We are interested in tracking a target position $\boldsymbol{x}_{t}=\left(x_{1, t}, x_{2, t}\right)^{T}$ at each instant $t(t=1, \ldots, N$, where $N$ denotes the number of observations). Consider the activated sensor $i$, its observation $\gamma_{t}^{i}$ is modeled by:

$$
\gamma_{t}^{i}=K\left\|\boldsymbol{x}_{t}-\boldsymbol{s}^{i}\right\|^{\eta}+\epsilon_{t}
$$

where $\epsilon_{t}$ is a Gaussian noise with zero mean and known variance $\sigma_{\epsilon}^{2}$. The constants $\eta$ and $K$ are also assumed to be known. The sensor transmits its observation to the cluster head $(\mathrm{CH})$ only 
if the target is detected, which is equivalent to the condition that $R_{\min } \leq\left\|\boldsymbol{x}_{t}-\boldsymbol{s}^{i}\right\| \leq R_{\max }$ where $R_{\max }$ (resp. $R_{\min }$ ) denotes the maximum (resp. minimum) distance at which the sensor can detect the target. Based on gathered transmitted sensor information, the cluster head is in charge of processing data in order to track the target. In order to save energy, before being transmitted, the observation is quantized by partitioning the observation space into $N_{t}^{i}$ intervals $\mathcal{R}_{j}=\left[\tau_{j}, \tau_{j+1}\right]$, where $j \in\left\{1, \ldots, N_{t}^{i}\right\}$. The number $N_{t}^{i}=2^{L_{t}^{i}}$ denotes the quantization level.

The quantizer is assumed to have an uniform step $\Delta=\frac{\tau_{N_{t}^{i}+1}-\tau_{1}}{N_{t}^{i}}$, with the initial and the last thresholds set to $\tau_{1}=K R_{\min }^{\eta}-\sigma_{\epsilon}$ and $\tau_{N_{t}^{i}+1}=K R_{\max }^{\eta}+\sigma_{\epsilon}$, respectively. The quantization rule is then given by:

$$
y_{t}^{i}=Q\left(\gamma_{t}^{i}\right)=d_{j} \text { if } \gamma_{t}^{i} \in\left[\tau_{j}(t), \tau_{j+1}(t)\right]
$$

where, the normalized $d_{j}$ is given by $d_{j}=\frac{\tau_{j}(t)+\frac{\Delta}{2}}{\tau_{N_{t+1}}(t)-\tau_{1}(t)}$, and $Q()$ is the quantization function. Figure 10 depicts a simple example for the quantized observation model.

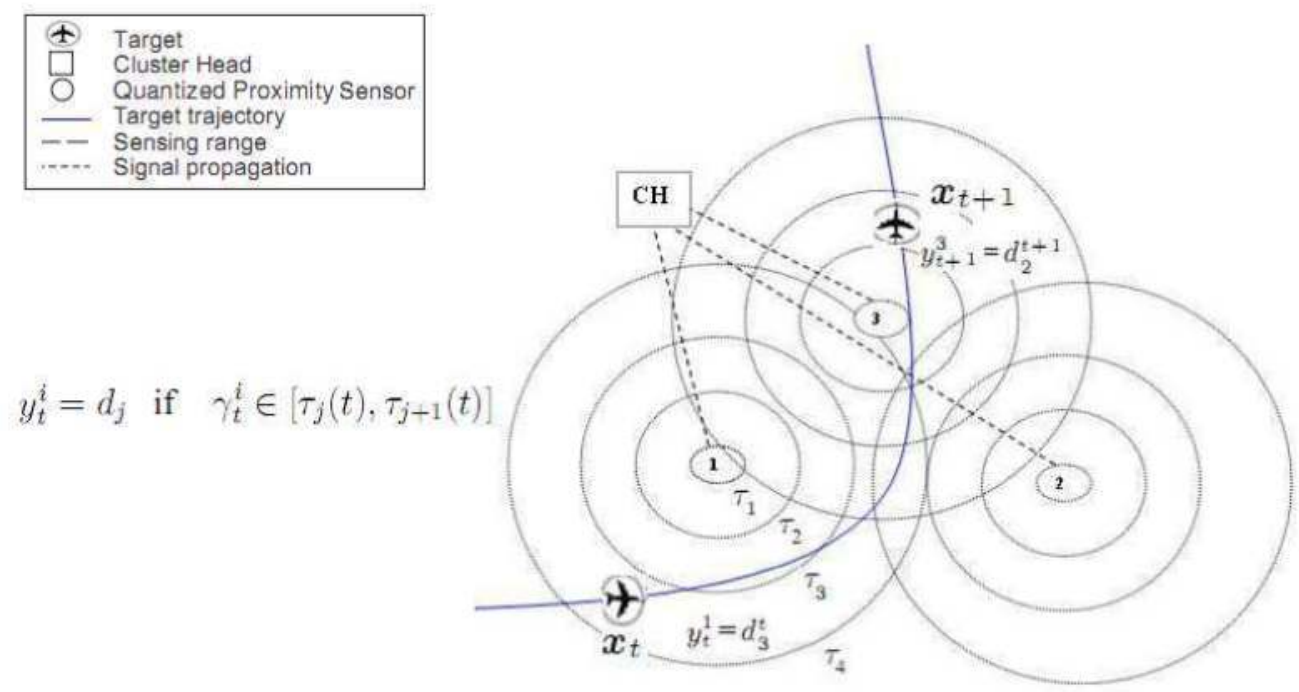

Fig. 10. The quantized observation model is described by a simple example. With respect to the first sensor, the target is within its sensing range at instant $t$. Observation $y_{t}^{1}$ is thus transmitted to the $\mathrm{CH}$. However the second sensor keeps silent. The situation at instant $\mathrm{t}+1$ can be similarly deduced.

Then, the signal received by the $\mathrm{CH}$ from the sensor $i$ at the sampling instant $t$ is written as,

$$
z_{t}^{i}=\beta_{t}^{i} \cdot y_{t}^{i}+n_{t}
$$


where $\beta_{t}^{i}=r_{i}^{\lambda}$ is the $i$-th sensor channel attenuation coefficient at the sampling instant $t, r_{i}$ is the transmission distance between the $i$-th sensor and the $\mathrm{CH}, \lambda$ is the path-loss exponent and $n_{t}$ is a random Gaussian noise with a zero mean and a known variance $\sigma_{n}^{2}$. Figure 11 summarizes the transmission scheme occurring during the data processing.

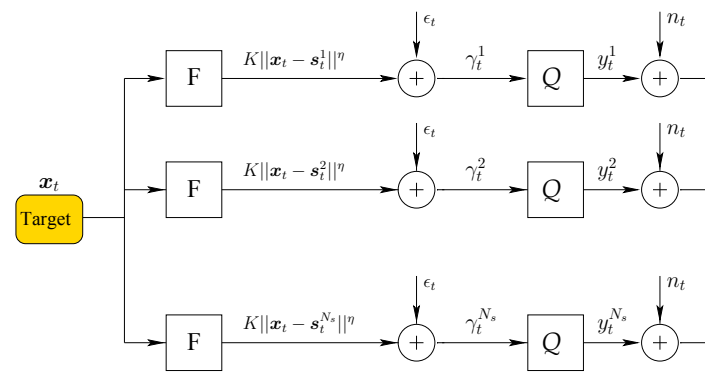

Fig. 11. Illustration of the communications path-ways in a WSN: The $1^{\text {st }}$ sensor makes a noisy reading $\gamma_{t}^{1}$. The quantized measurement $y_{t}^{1}=Q\left(\gamma_{t}^{1}\right)$ with $L_{t}^{1}$ bits of precision is sent to the $\mathrm{CH}$. The measurement $z_{t}^{1}$ is received by the $\mathrm{CH}$, it is corrupted by an additive white Gaussian noise $n_{t}$.

The next section is devoted to the mutual information parameter computing.

\subsection{Parameters that measure the information relevance of sensor measurements}

The main idea of these parameters is to define the basic parameters that may influence the relevance of the sensors cooperation, which are: (1) information content that can be transferred from candidate sensor $i ; \boldsymbol{M I}\left(\boldsymbol{x}_{t}, z_{t}^{i}\right)$ (detailed in 4.2.1, (2) the Fisher information matrix; $\boldsymbol{F I}\left(\boldsymbol{x}_{t}, z_{t}^{i}\right)$ (detailed in 4.2.2) and the Kullback Leibler distance (KLD), which is detailed in 4.2.3.

\subsubsection{Computation of the Mutual Information function}

The mutual information function is often used to measure the efficiency of a given information. The $M I$ function is a quantity measuring the amount of information that the observable variable $z_{t}$ carries about the unknown parameter $\boldsymbol{x}_{t}$. The mutual information between the observation $z_{t}^{i}$ and the source $\boldsymbol{x}_{t}$ is proportional to Mansouri et al. (2009):

$$
M I\left(\boldsymbol{x}_{t}, z_{t}^{i}\right) \propto p\left(z_{t}^{i} \mid \boldsymbol{x}_{t}\right) \log \left(p\left(z_{t}^{i} \mid \boldsymbol{x}_{t}\right)\right)
$$

The likelihood function $(L)$ is expressed as,

$$
L\left(\boldsymbol{s}^{i}\right)=p\left(z_{t}^{i} \mid \boldsymbol{x}_{t}\right)=\sum_{j=0}^{N_{t}^{i}-1} p\left(\tau_{j}(t)<\gamma_{t}^{i}<\tau_{j+1}(t)\right) \mathcal{N}\left(h_{t}^{i} d_{j}, \sigma_{\epsilon}^{2}\right)
$$


where

$$
p\left(\tau_{j}(t)<\gamma_{t}^{i}<\tau_{j+1}(t)\right)=\int_{\tau_{j}(t)}^{\tau_{j+1}(t)} \mathcal{N}\left(\rho_{\gamma_{t}^{i}}\left(s^{i}\right), \sigma_{n}^{2}\right) d \gamma_{t}
$$

is computed according to the quantization rule defined in (5), in which

$$
\rho_{\gamma_{t}^{i}}\left(s^{i}\right)=K\left\|\boldsymbol{x}_{t}-s^{i}\right\|^{\eta}
$$

\subsubsection{Fisher information matrix}

The fisher information (FI) matrix is a quantity measuring the amount of information that the observable variable $z_{t}^{i}$ carries about the unknown parameter $\boldsymbol{x}_{t}$. The FI matrix elements at the sampling instant $t$ are given by:

$$
\begin{aligned}
& {\left[F I\left(\boldsymbol{x}_{t}, \boldsymbol{s}^{i}, N_{t}^{i}\right)\right]_{l, k}=} E_{z_{t}^{i} \mid \boldsymbol{x}_{t}}\left[\frac{\partial \log \left(p\left(z_{t}^{i} \mid \boldsymbol{x}_{t}\right)\right)}{\partial \boldsymbol{x}_{(l, t)}} \frac{\partial \log \left(p\left(z_{t}^{i} \mid \boldsymbol{x}_{t}\right)\right)}{\partial \boldsymbol{x}_{(k, t)}}\right] \\
&(l, k) \in\{1,2\} \times\{1,2\}
\end{aligned}
$$

where $z_{t}^{i}$ denotes the observation of the $i$-th sensor at the sampling instant $t, \boldsymbol{x}_{t}=\left[x_{1}, x_{2}\right]^{T}$ is the unknown $2 \times 1$ vector to be estimated, and $E_{z_{t}^{i} \mid \boldsymbol{x}_{t}}[$.$] denotes the expectation with respect$ the likelihood function $p\left(\boldsymbol{z}_{t} \mid \boldsymbol{x}_{t}\right)$, which is given by

$$
p\left(z_{t}^{i} \mid \boldsymbol{x}_{t}\right)=\sum_{j=0}^{N_{t}^{i}-1} p\left(\tau_{j}(t)<\gamma_{t}^{i}<\tau_{j+1}(t)\right) \mathcal{N}\left(\beta d_{j}, \sigma_{\epsilon}^{2}\right)
$$

Then, the derivative of the log-likelihood function can be expressed as,

$$
\begin{array}{r}
\frac{\partial \log \left(p\left(z_{t}^{i} \mid \boldsymbol{x}_{t}\right)\right)}{\partial \boldsymbol{x}_{l, t}}=\frac{\eta K}{\sqrt{2 \sigma_{n}^{2}}}\left(x_{l, t}-s_{l, i}\right)\left\|x_{l, t}-s_{l, i}\right\|^{\eta-2} \times \sum_{k=1}^{N_{t}^{i}}\left[\exp \left(-\frac{1}{2} \frac{\left(\tau_{k}-\rho_{\gamma_{t}^{i}}\left(\boldsymbol{x}_{t}\right)\right)^{2}}{\sigma_{n}^{2}}\right)-\right. \\
\left.\exp \left(-\frac{1}{2} \frac{\left(\tau_{k+1}-\rho_{\gamma_{t}^{i}}\left(\boldsymbol{x}_{t}\right)\right)^{2}}{\sigma_{n}^{2}}\right)\right] \times \exp \left(-\frac{1}{2} \frac{\left(z_{t}(k)-d_{k}\right)^{2}}{\sigma_{\epsilon}^{2}}\right) / \sum_{k=1}^{N_{t}^{i}}\left[\operatorname{erfc}\left(\frac{\tau_{k}-\rho_{\gamma_{t}^{i}}\left(\boldsymbol{x}_{t}\right)}{\sqrt{2 \sigma_{n}^{2}}}\right)-\right. \\
\left.\operatorname{erfc}\left(\frac{\tau_{k+1}-\rho_{\gamma_{t}^{i}}\left(\boldsymbol{x}_{t}\right)}{\sqrt{2 \sigma_{n}^{2}}}\right)\right] \times \exp \left(-\frac{1}{2} \frac{\left(z_{t}(k)-d_{k}\right)^{2}}{\sigma_{\epsilon}^{2}}\right)
\end{array}
$$

Substituting expression (13) in (11), the FI matrix is easily computed by integrating over the likelihood function $p\left(z_{t}^{i} \mid \boldsymbol{x}_{t}\right)$ at the sampling instant $t$. 


\subsubsection{Computing of the Kullback Leibler distance (KLD)}

In certain problems, we would like to measure the distance between two statistical models. For example, this distance can be used in evaluating the training algorithm or classifying the estimated models Juang \& Rabiner (1985). The Kullback-Leibler distance or the relative entropy arises in many contexts as an appropriate measurement of the distance between two distributions. The KLD between the two probability density functions $p$ and $\widehat{p}$ is defined as Cover \& Thomas (2006):

$$
K L D(p \| \widehat{p})=\int p \log \frac{p}{\hat{p}}
$$

For hidden Markov models, the distribution function is very complex, and practically it can be only computed via a recursive procedure; the "forward/backward" or "upward/downward" algorithms Rabiner (1989); Ronen et al. (1995). Thus there is no simple closed form expression for the KLD for these models. Commonly, the Monte-Carlo method is used to numerically approximate the integral in (14) as:

$$
K L D(p \| \widehat{p})=\boldsymbol{E}_{p}(\log (p)-\log (\widehat{p}))
$$

\section{5. conclusion}

In this chapter, we have studied the parameters that may influence the performance of the WSN. We have started by the sensor nodes characteristics as battery, processor speed, storage capacity and radio communication. The sensor nodes types have been classified according to the probable applications as terrestrial, underground, underwater and multimedia. Indeed, the needed sensor node characteristics change from an application to another, as e.g., the high communication capacity needed in underwater applications to deal with the acoustic signal propagation problems, the battery optimization in the context of large scale terrestrial application, or the storage and processing problems to treat the captured images, videos and sounds in a multimedia WSN. In addition, the simulations have shown the importance of adjusting the transmitting power of the sensor nodes to reduce the estimation error in target tracking while maintaining the power consumption of the sensor nodes.

Later on, we have discussed the impact of the network deployment on the WSNs' performance, in terms of data accuracy and optimal lifetime maximization. This chapter has focused mainly on the case of random distribution/deployment of nodes, as the pre-planed deployments are generally adapted to some performance levels. We have shown through successive simulations the importance of the network density on reducing the distance estimation error, in the context of multi objects tracking. The simulations have proved also, the importance of taking into consideration, in any proposal, the position of each sensor nodes within the network. E.g., by applying special behaviors to sensor nodes in critical positions, we can maximize the occurred duration before the first network partitioning, which could help to optimally maximize the WSN lifetime.

Finally, this chapter has dedicated an important part to the processing of the information that we may have in the WSN. We have studied the parameters that could help to measure the relevance of the sensor nodes measurement. From these parameters, we have detailed the computation of the mutual information function, the fisher information matrix and the computation of the Kullback Leibler distance. We have also presented a computation model related to these parameters, which is the quantized observation model. 


\section{References}

Akyildiz, I. F., Melodia, T. \& Chowdhury, K. R. (2007). A survey on wireless multimedia sensor networks, Comput. Netw. 51(4): 921-960.

Akyildiz, I. F., Su, W., Sankarasubramaniam, Y. \& Cayirci, E. (2002). Wireless sensor networks: a survey, The International Journal of Computer and Telecommunications Networking 38(4): 393-422.

Baronti, P., Pillai, P., Chook, V. W., Chessa, S., Gotta, A. \& Hu, F. Y. (2007). Wireless sensor networks: A survey on the state of the art and the 802.15.4 and zigbee standards, Computer Communications 30(7): 1655-1695.

URL: $h$ ttp://dx.doi.org/10.1016/j.comcom.2006.12.020

Chang, J.-H. \& Tassiulas, L. (2004). Maximum lifetime routing in wireless sensor networks, IEEE/ACM Trans. Netw. 12(4): 609-619.

Chatzigiannakis, I., Kinalis, A. \& Nikoletseas, S. (2008). Efficient data propagation strategies in wireless sensor networks using a single mobile sink, Comput. Commun. 31(5): 896914.

Cheng, L., Chen, Y., Chen, C. \& Ma, J. (2009). Query-based data collection in wireless sensor networks with mobile sinks, IWCMC '09: Proceedings of the 2009 International Conference on Wireless Communications and Mobile Computing, ACM, New York, NY, USA, pp. 1157-1162.

Cover, T. \& Thomas, J. (2006). Elements of information theory, John Wiley and sons.

Cui, S., Goldsmith, A. \& Bahai, A. (2005). Energy-constrained modulation optimization, IEEE Transactions on Wireless Communications 4(5): 2349-2360.

Djuric, P., Kotecha, J., Jianqui, Z., Yufei, H., Ghirmai, T., Bugallo, M. \& Miguez, J. (2003). Particle filtering, IEEE Signal Processing Magazine 20(5): 19-38.

Gay, D., Welsh, M., Levis, P., Brewer, E., von Behren, R. \& Culler, D. (2003). The nesc language: A holistic approach to networked embedded systems, In Proceedings of Programming Language Design and Implementation (PLDI, pp. 1-11.

Heidemann, J., Ye, W., Wills, J., Syed, A. \& Li, Y. (2006). Research challenges and applications for underwater sensor networking, Proceedings of the IEEE Wireless Communications and Networking Conference, IEEE, Las Vegas, Nevada, USA, pp. 228-235.

Hill, J., Szewczyk, R., Woo, A., Hollar, S., Culler, D. \& Pister, K. (2000). System architecture directions for networked sensors, SIGPLAN Not. 35(11): 93-104.

Ian F., A. \& Erich P., S. (2006). Wireless underground sensor networks:research challenges, $A d$ Hoc Networks 4: 669-686.

Juang, B. \& Rabiner, L. (1985). A probabilistic distance measure for hidden Markov models, ATET Bell Laboratories technical journal 64(2): 391-408.

Li, M. \& Liu, Y. (2007). Underground structure monitoring with wireless sensor networks, IPSN '07: Proceedings of the 6th international conference on Information processing in sensor networks, pp. 69-78.

Mansouri, M., Ouchani, I., Snoussi, H. \& Richard, C. (2009). Cramer-Rao Bound-based adaptive quantization for target tracking in wireless sensor networks, IEEE Workshop on Statistical Signal Processing (SSP).

Mansouri, M., Snoussi, H. \& Richard, C. (n.d.). Robust target tracking with quantized proximity sensors, 2010,IEEE International Symposium on Wireless Pervasive Computing (ISWPC).

Ok, C.-S., Lee, S., Mitra, P. \& Kumara, S. (2009). Distributed energy balanced routing for wireless sensor networks, Comput. Ind. Eng. 57(1): 125-135. 
Platon, E. \& Sei, Y. (2008). Security software engineering in wireless sensor networks., Progress in Informatics .

Polastre, J., Hill, J. \& Culler, D. (2004). Versatile low power media access for wireless sensor networks, SenSys '04: Proceedings of the 2nd international conference on Embedded networked sensor systems, ACM, New York, NY, USA, pp. 95-107.

Pompili, D., Melodia, T. \& Akyildiz, I. F. (2006). Deployment analysis in underwater acoustic wireless sensor networks, WUWNet '06: Proceedings of the 1st ACM international workshop on Underwater networks, pp. 48-55.

Rabiner, L. (1989). A tutorial on hidden Markov models and selected applications inspeech recognition, Proceedings of the IEEE 77(2): 257-286.

Ronen, O., Rohlicek, J. \& Ostendorf, M. (1995). Parameter estimation of dependence tree models using the EM algorithm, IEEE Signal Processing Letters 2(8): 157-159.

Sardouk, A., Rahim-Amoud, R., Merghem-Boulahia, L. \& Gaiti, D. (2011). Power-aware agentsolution for information communication in wsn, Telecommunication Systems 48. To appear.

Shaylor, N., Simon, D. N. \& Bush, W. R. (2003). A java virtual machine architecture for very small devices, SIGPLAN Not. 38(7): 34-41.

Sohraby, K., Minoli, D. \& Znati, T. (2007). Wireless Sensor Networks, Technology, Protocols, and Applications, WILLEY.

Stavros, T. \& Leandros, T. (2006). Optimal deployment of large wireless sensor networks, IEEE Transactions on Information Theory 52(7): 2935-2953.

Sun (2008). Sun ${ }^{T M}$ Small Programmable Object Technology (Sun SPOT) Theory of Operation, Technical report, Sun Microsystem, Sun Labs.

Ye, W., Heidemann, J. \& Estrin, D. (2002). An energy-efficient mac protocol for wireless sensor networks.

URL: citeseer.ist.psu.edu/ye01energyefficient.html

Yick, J., Mukherjee, B. \& Ghosal, D. (2008). Wireless sensor network survey, Computer Networks 52(12): 2292-2330. 


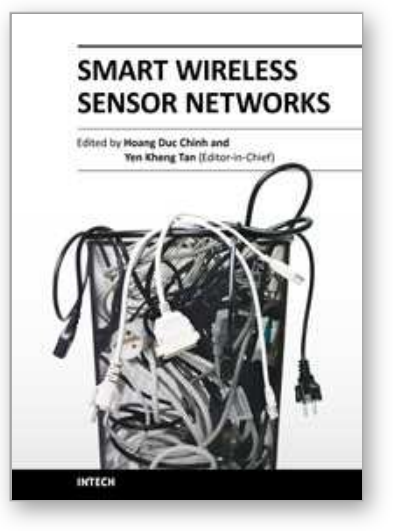

\author{
Smart Wireless Sensor Networks \\ Edited by Yen Kheng Tan
}

ISBN 978-953-307-261-6

Hard cover, 418 pages

Publisher InTech

Published online 14, December, 2010

Published in print edition December, 2010

The recent development of communication and sensor technology results in the growth of a new attractive and challenging area â€" wireless sensor networks (WSNs). A wireless sensor network which consists of a large number of sensor nodes is deployed in environmental fields to serve various applications. Facilitated with the ability of wireless communication and intelligent computation, these nodes become smart sensors which do not only perceive ambient physical parameters but also be able to process information, cooperate with each other and self-organize into the network. These new features assist the sensor nodes as well as the network to operate more efficiently in terms of both data acquisition and energy consumption. Special purposes of the applications require design and operation of WSNs different from conventional networks such as the internet. The network design must take into account of the objectives of specific applications. The nature of deployed environment must be considered. The limited of sensor nodesâ€ $€^{\mathrm{TM}}$ resources such as memory, computational ability, communication bandwidth and energy source are the challenges in network design. A smart wireless sensor network must be able to deal with these constraints as well as to guarantee the connectivity, coverage, reliability and security of networkâ $€^{\mathrm{TM}} \mathbf{S}$ operation for a maximized lifetime. This book discusses various aspects of designing such smart wireless sensor networks. Main topics includes: design methodologies, network protocols and algorithms, quality of service management, coverage optimization, time synchronization and security techniques for sensor networks.

\title{
How to reference
}

In order to correctly reference this scholarly work, feel free to copy and paste the following:

Majdi Mansouri, Ahmad Sardouk, Leila Merghem-boulahia, Dominique Gaiti, Hichem Snoussi, Rana Rahimamoud and Cedric Richard (2010). Factors that May Influence the Performance of Wireless Sensor Networks, Smart Wireless Sensor Networks, Yen Kheng Tan (Ed.), ISBN: 978-953-307-261-6, InTech, Available from: http://www.intechopen.com/books/smart-wireless-sensor-networks/factors-that-may-influence-theperformance-of-wireless-sensor-networks

\section{INTECH}

open science | open minds

\author{
InTech Europe \\ University Campus STeP Ri \\ Slavka Krautzeka 83/A \\ 51000 Rijeka, Croatia
}

\author{
InTech China \\ Unit 405, Office Block, Hotel Equatorial Shanghai \\ No.65, Yan An Road (West), Shanghai, 200040, China \\ 中国上海市延安西路 65 号上海国际贵都大饭店办公楼 405 单元
}


Phone: +385 (51) 770447

Fax: +385 (51) 686166

www.intechopen.com
Phone: +86-21-62489820

Fax: +86-21-62489821 
(C) 2010 The Author(s). Licensee IntechOpen. This chapter is distributed under the terms of the Creative Commons Attribution-NonCommercialShareAlike-3.0 License, which permits use, distribution and reproduction for non-commercial purposes, provided the original is properly cited and derivative works building on this content are distributed under the same license. 